Journal of Algebraic Combinatorics 5 (1996), 337-351

(C) 1996 Kluwer Academic Publishers. Manufactured in The Netherlands.

\title{
Free Lie Superalgebras, Trees and Chains of Partitions
}

\author{
GUY MELANÇON* \\ LABRI, 351, Cours de la Libération, Université de Bordeaux I, 33405 Talence Cedex, France \\ CHRISTOPHE REUTENAUER* \\ Université du Québec à Montréal, Département de mathématiques, C.P. 8888, Succursale Centre-ville, Montréal \\ (Québec) H3C 3P8, Canada
}

Received December 2, 1994

Keywords: Lie, superalgebra, free, combinatorics, construction

\section{Introduction}

Several representations of the symmetric group, arising from different combinatorial, algebraic and geometric constructions, have lead to the same character, up to multiplication by the sign character: the homology of partition lattice (cf. $[5,7,13])$, the top component of a special quotient of the Stanley-Reisner ring of this same lattice [4], the top component of the cohomology algebra of the variety $\left\{x \in \mathbb{C}^{n} \mid x_{i} \neq x_{j}\right.$ if $\left.i \neq j\right\}$ computed by Arnold [9], the free Lie algebra [7, 8]. Barcelo [1] and Bergeron and Barcelo [2] have also proved this equality of characters by showing that the matrices of these representations in the classical bases (Lyndon basis of the free Lie algebra, Garsia-Stanton basis, NBC basis of Björner [3]) are equal, up to sign-character and transposition. The latter work has been the starting motivation of the present paper.

It turns out that the character of $S_{n}$ acting on the (multilinear part of the) free Lie algebra is the product by the sign character by its character on the (oddly generated) free Lie superalgebra $\mathcal{R}(X)$. This fact is already implicit in Ree's paper [12]. In the present paper, we give several combinatorial/algebraic constructions (analytic functors [7], or polynomial functors [10]) which are variant of the classical construction of $\mathcal{R}(X)$ by trees (representing the brackets) or of its dual; these different functors coincide in their multilinear part with the previously mentioned constructions on the partition lattice, so that the equality of characters and matrices becomes natural.

A striking fact in all these constructions is that they are obtained by introducing relations which in all cases are of two kinds: one of length 2, and one of length 3 (antisymmetry and Jacobi identity for the free Lie superalgebra, cohomology or Garsia-Stanton relations in the Stanley-Reisner ring, antisymmetry and cyclicity in the Arnold algebra). At first glance,

*The authors were supported by NSERC (Canada). 
there should be strict correspondence between these relations in all these constructions, since they provide the same character. This is however not the case, and our work shows how one passes from one to another set of relations.

The different constructions of $\mathcal{R}(X)$ (or its dual) that we give rest all on several kinds of trees, familiar to combinatorialists and computer scientists, and mappings among them. They are all labelled (nodes, leafs, edges) in $X$ and $1,2,3, \ldots$ One example are the spatial rooted complete binary trees, with $X$-labelled leafs and nodes labelled $1,2,3, \ldots$; in the multilinear case, they are in bijection with maximal chains of partitions of $X$ (cf. [14]).

This paper extends and corrects [11], and an earlier version, for which we gratefully acknowledge the two referees. We thank also Claudia Malvenuto, for preliminary discussions, and Sheila Sundaram for explaining us the links between homology and Garsia-Stanton relations of the partition lattice. During the time of preparation of this article, starting 1991, through [11] and the previous version entitled "The Free Lie Superalgebra and Representations of the Symmetric Group", there has been some overlap with a work of Michelle Wachs ([14] and her talk at the Jerusalem Combinatorics Conference in May, 1993). Especially, Corollary 3.2 has been found independently by her, and she also uses the previously mentioned bijection. We think however that our approach is justified, among others because we consider the non multilinear case, construct combinatorially the free Lie superalgebra and its dual, thereby relating the whole thing to the work of [9]. A related work is also [6].

Recall that the (oddly generated) free Lie superalgebra on $X$ over $\mathbb{Q}$ is the quotient of the free (nonassociative, noncommutative) algebra $\mathcal{A}(X)$ by the relations (called antisymmetry and Jacobi relations)

$$
\begin{aligned}
{[P, Q]+(-1)^{p q}[Q, P] } & =0, \\
{[P,[Q, R]] } & =[[P, Q], R]+(-1)^{p q}[Q,[P, R]],
\end{aligned}
$$

where the product is denoted by brackets, and where $P, Q, R$ are homogeneous elements with degrees $p, q, r$. By a result of Ree [12], $\mathcal{R}(X)$ may be identified with the sub Lie superalgebra of the free associative algebra $\mathbb{Q}\langle X\rangle$ generated by $X$, where the bracket is $[P, Q]=P Q-(-1)^{p q} Q P$ for any homogeneous polynomials $P, Q$ in $\mathbb{Q}\langle X\rangle$. We call an element of $\mathcal{R}(X)$ a Ree polynomial.

\section{Planar rooted binary trees}

An element of $T(X)$ will be a planar, rooted, binary and complete tree $t$, with leaves labelled in $X$, with internal nodes having distinct labels in $\{1,2, \ldots,|t|-1\}$, where $|t|$ is the $X$ degree of $t$ (number of leaves), and where these labels increase from leaves to the root. The space $\mathcal{T}(X)$ is the $\mathbb{Q}$-vector space with basis $T(X)$.

Let $L(X)$ denote the set of words of the form $x_{1} i_{1} x_{2} i_{2} \cdots i_{n-1} x_{n}$, where $n \geq 1, x_{j} \in X$ and $\left\{i_{1}, \ldots, i_{n-1}\right\}=\{1,2, \ldots, n-1\}$. There is a natural bijection between $L(X)$ and $T(X)$, described in figure 1 , and obtained by projecting the tree onto an horizontal line. This allows to associate to each tree $t$ in $T(X)$ a permutation $\sigma$, by forgetting in the corresponding word the letters in $X$; then we define a natural surjective linear mapping $f^{\prime}: \mathcal{T}(X) \rightarrow \mathcal{R}(X)$ recursively by $f^{\prime}(x)=x$ if $x \in X, f^{\prime}(t)=\left[f^{\prime}\left(t_{1}\right), f^{\prime}\left(t_{2}\right)\right]$ if $t_{1}\left(t_{2}\right)$ is the left (right) subtree of $t$; that is, $f^{\prime}(t)$ is the element of $\mathcal{R}(X)$ corresponding to the bracketting determined by 


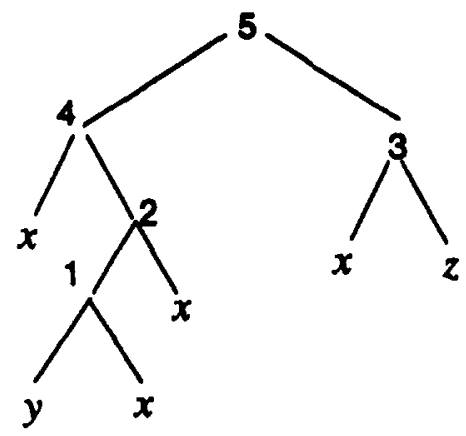

$x 4 y 1 \times 2 \times 5 \times 3 z$

Figure 1.

the tree $t$; in figure $1, f^{\prime}(t)$ is $[[x,[[y, x], x]],[x, z]]$. Then we define the surjective linear mapping $f: \mathcal{T}(X) \rightarrow \mathcal{R}(X)$ by $f(t)=(-1)^{\sigma} f^{\prime}(t)$, where $\sigma$ is the permutation of $t$, and $(-1)^{\sigma}$ its signature.

Define a subspace $\mathcal{T}^{\prime}(X)$ of $\mathcal{T}(X)$, spanned by the elements shown in figure 2 , where dots indicate that the trees are otherwise equal. We define a product [, ] on $\mathcal{T}(X)$ by the following rule. Let $t_{1}, t_{2} \in \mathcal{T}(X)$ and denote by $\bar{t}_{2}$ the tree obtained by shifting the internal labels of $t_{2}$ from $\left\{1, \ldots,\left|t_{2}\right|-1\right\}$ to $\left\{\left|t_{1}\right|, \ldots,\left|t_{1}\right|+\left|t_{2}\right|-2\right\}$; then the product $\left[t_{1}, t_{2}\right]$ of $t_{1}$ and $t_{2}$ is $(-1)^{\left|t_{2}\right|-1} t$, where the tree $t$ has $t_{1}$ as left subtree, $\bar{t}_{2}$ as right subtree and $\left|t_{1}\right|+\left|t_{2}\right|-1$ as root label. See figure 3 .

Theorem 2.1 The product in $\mathcal{T}(X)$ defines a structure of super Lie algebra on $\mathcal{T}(X) / \mathcal{T}^{\prime}$ $(X)$, which is isomorphic with $\mathcal{R}(X)$, the free super Lie algebra.

At a first glance, this result seems trivial, since $\mathcal{R}(X)$ is defined as a quotient of the space spanned by trees (not internally labelled) by the super antisymmetry and Jacobi relations, and since the product in $\mathcal{T}(X)$ is close to that of $\mathcal{R}(X)$. However, there is a technical difficulty, solved by the next lemma, whose proof is deferred at the end of the paper.

Lemma 2.2 The product in $\mathcal{T}(X)$ satisfies, mod. $\mathcal{T}^{\prime}(X)$, the super antisymmetry and Jacobi relations.

Proof of Theorem 2.1: If $t_{1} \equiv t_{1}^{\prime} \bmod . \mathcal{T}^{\prime}(X)$, then clearly $\left[t_{1}, t_{2}\right] \equiv\left[t_{1}^{\prime}, t_{2}\right] \bmod . \mathcal{T}^{\prime}(X)$, and similarly on the other side. This shows that the product is well-defined in $\mathcal{T}(X) / \mathcal{T}^{\prime}(X)$ and Lemma 2.2 implies that $\mathcal{T}(X) / \mathcal{T}^{\prime}(X)$ is a super Lie algebra, which is clearly generated by $X$.

We verify that $f: \mathcal{T}(X) \rightarrow \mathcal{R}(X)$ is a homomorphism. Indeed, we have $f\left(\left[t_{1}, t_{2}\right]\right)=$ $f\left((-1)^{\left|t_{2}\right|-1} t\right)=(-1)^{\left|t_{2}\right|-1}(-1)^{\sigma} f^{\prime}(t)$ on one hand, and on the other $\left[f\left(t_{1}\right), f\left(t_{2}\right)\right]=$ $\left[(-1)^{\sigma_{1}} f^{\prime}\left(t_{1}\right),(-1)^{\sigma_{2}} f^{\prime}\left(t_{2}\right)\right]=(-1)^{\sigma_{1}}(-1)^{\sigma_{2}}\left[f^{\prime}\left(t_{1}\right), f^{\prime}\left(t_{2}\right)\right]=(-1)^{\sigma_{1}}(-1)^{\sigma_{2}} f^{\prime}(t)$, and we conclude, in view of the following fact:

The permutation $\sigma$ associated with $t$ is $\sigma_{1}(n-1) \bar{\sigma}_{2}$, where $n=|t|$ and $\bar{\sigma}_{2}$ is obtained from $\sigma_{2}$ by adding $n_{1}-1$ to its digits. Moreover, we have $(-1)^{\sigma}=(-1)^{n_{2}-1}(-1)^{\sigma_{1}}(-1)^{\sigma_{2}}$. 
(2.3)

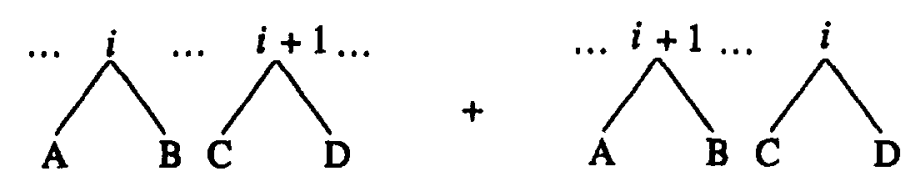

(2.4)
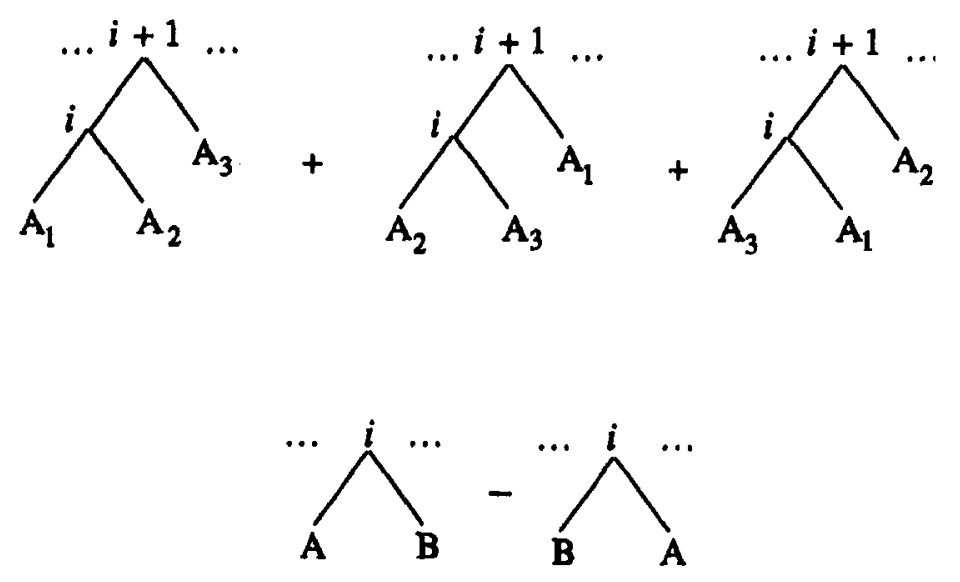

Figure 2.

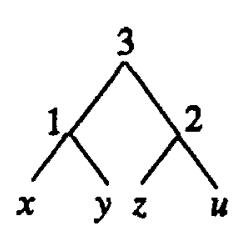

$t_{1}$

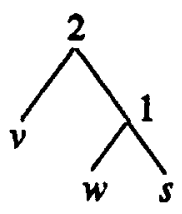

$t_{2}$

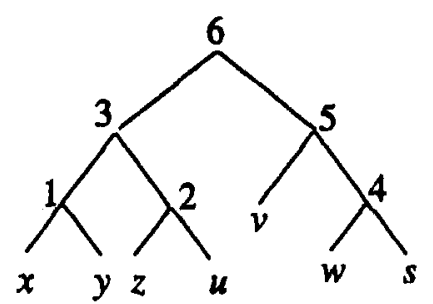

$t_{x}$

Figure 3. 
We verify now that $\operatorname{Ker} f \supseteq \mathcal{T}^{\prime}(X)$. This will imply that $f$ defines a super Lie algebra homomorphism $\mathcal{T}(X) / \mathcal{T}^{\prime}(X) \rightarrow \mathcal{R}(X)$; since $\mathcal{T}(X) / \mathcal{T}^{\prime}(X)$ is generated by $X$ and since $\mathcal{R}(X)$ is free, it must be an isomorphism.

Clearly, element (2.3) is in Ker $f$, since the two trees are identical when they lose their internal labels, and have permutations of opposite sign. For (2.4), let $a_{1}, a_{2}, a_{3}$ be the corresponding degrees; the permutations associated to the three trees are $u \alpha_{1} i \alpha_{2}(i+1)$ $\alpha_{3} v, u \alpha_{2} i \alpha_{3}(i+1) \alpha_{1} v$ and $u \alpha_{3} i \alpha_{1}(i+1) \alpha_{2} v$, where $\alpha_{1}, \alpha_{2}, \alpha_{3}$ are the permutations of $A_{1}, A_{2}, A_{3}$, of lengths $a_{1}-1, a_{2}-1, a_{3}-1$, and where $i, i+1$ are greater than the digits in $\alpha_{1}, \alpha_{2}, \alpha_{3}$. A simple verification, left to the reader, shows that their signatures are $\varepsilon(-1)^{a_{3} a_{1}}$, $\varepsilon(-1)^{a_{1} a_{2}}, \varepsilon(-1)^{a_{2} a_{3}}$ with $\varepsilon= \pm 1$. Denoting $A_{i}^{\prime}=f^{\prime}\left(A_{i}\right)$, we must therefore show that $(-1)^{a_{3} a_{1}}\left[\left[A_{1}^{\prime}, A_{2}^{\prime}\right], A_{3}^{\prime}\right]+(-1)^{a_{1} a_{2}}\left[\left[A_{2}^{\prime}, A_{3}^{\prime}\right], A_{1}^{\prime}\right]+(-1)^{a_{2} a_{3}}\left[\left[A_{3}^{\prime}, A_{1}^{\prime}\right], A_{2}^{\prime}\right]=0$ in $\mathcal{R}(X)$. But this is a consequence of (1.1) and (1.2). For (2.5), a similar argument shows that this element is in $\operatorname{Ker} f$, as a consequence of (1.1).

For two trees $t^{\prime}, t$ in $T(X)$ we write $t^{\prime} \sim t$ if $t^{\prime}$ is obtained from $t$ by iteratively twisting $t$ around some of its internal nodes. This is clearly an equivalence relation. We denote $w(t)$ the word of $t$ in $X^{*}$, obtained by forgetting in the corresponding word the digits $1,2, \ldots$ In figure 1 , it is $x y x x x z$.

Corollary 2.3 Let $\mathcal{R}(X)$ be embedded in $\mathbb{Q}\langle X\rangle$. Then the isomorphism of Theorem 2.1 sends each tree t onto $\sum_{t^{\prime} \sim t}(-1)^{\sigma^{\prime}} w\left(t^{\prime}\right)$, where $\sigma^{\prime}$ is the permutation of $t^{\prime}$.

Proof: If $t^{\prime}$ is obtained from $t$ by twisting around the node labelled $i$ its subtrees $A$ and $B$, then the signature of the corresponding permutations changes by $(-1)^{a b-1}(a=|A|, b=$ $|B|)$. Thus, by the super antisymmetry relation, we have $f\left(t^{\prime}\right)=f(t)$. Thus, $t^{\prime} \sim t \Rightarrow$ $f\left(t^{\prime}\right)=f(t)$. But in $f(t), w(t)$ appears with sign $(-1)^{\sigma}$. Since $f(t)=\sum_{t^{\prime} \sim t} \pm w\left(t^{\prime}\right)$, the corollary follows.

\section{Spatial rooted binary trees and chains of partitions}

An element of $S(X)$ will be a spatial (nonplanar), rooted, binary and complete tree $t$, with leaves labelled in $X$ and nodes distinctly labelled in $\{1, \ldots,|t|-1\}$, increasing from leaves to the root. Denote by $\mathcal{S}(X)$ the $\mathbb{Q}$-vector space with basis $S(X)$. There is a natural surjective linear mapping $g: \mathcal{T}(X) \rightarrow \mathcal{S}(X)$, such that $g(t)$ is, for $t$ in $T(X)$, the nonplanar tree associated to $t$.

Let $S^{\prime}(X)$ denote the subspace of $S(X)$ spanned by the elements of the form (2.3) and (2.4) in figure 2, considered as nonplanar trees. Define a product in $\mathcal{S}(X)$, similarly to that in $\mathcal{T}(X):\left[t_{1}, t_{2}\right]=(-1)^{\left|t_{2}\right|-1} t$, where $t$ has the two immediate subtrees $t_{1}, t_{2}$.

Theorem 3.1 The product in $\mathcal{S}(X)$ defines a structure of super Lie algebra on $\mathcal{S}(X) / \mathcal{S}^{\prime}$ $(X)$, which is isomorphic with $\mathcal{R}(X)$, the free Lie superalgebra.

Proof: The subspace $\operatorname{Ker} g$ of $\mathcal{T}(X)$ is generated by the elements (2.5) in figure 2, so that Ker $g \subseteq \mathcal{T}^{\prime}$. Moreover, $\mathcal{S}^{\prime}=g\left(\mathcal{T}^{\prime}\right)$, hence $g^{-1}\left(\mathcal{S}^{\prime}\right)=\mathcal{T}^{\prime}+\operatorname{Ker} g=\mathcal{T}^{\prime}$. Thus $\mathcal{T} / \mathcal{T}^{\prime}=\mathcal{T}^{\prime} / g^{-1}\left(\mathcal{S}^{\prime}\right) \simeq \mathcal{S} / \mathcal{S}^{\prime}$. Since the product is well-defined on $\mathcal{S} / \mathcal{S}^{\prime}$, the theorem follows. 


\section{tree $t$}

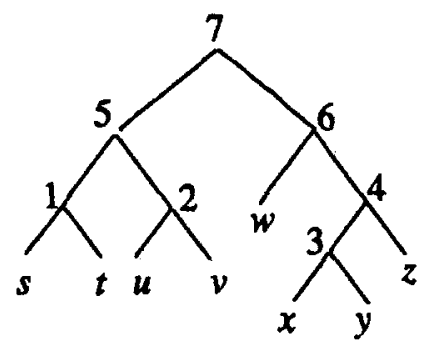

chain $h(t)$

\author{
$s|t| u|v| w|x| y \mid z$ \\ $s t|u| v|w| x|y| z$ \\ st $|u v| w|x| y \mid z$ \\ $s t|u v| w|x y| z$ \\ $s t|u v| w \mid x y z$ \\ $s t u v|w| x y z$ \\ $s t u v \mid w x y z$ \\ st $u v w \times y z$
}

Figure 4.

Remark Note that in [11], we have given a theorem analogue to Theorem 3.1, with $\mathcal{S} / \mathcal{S}^{\prime}$ replaced by a space constructed on maximal chains of partitions of multisets over $X$, subject to relations (3.1) and (3.2). This result is however not correct (although we obtain a strange Lie superalgebra which should be studied elsewhere); the reason is that the latter functor is not analytic in the sense of Joyal [7]. This can be seen for example on the chain $x y, x y \mid x y$, $x y y \mid x y$ which can be obtained by specialization from the two multilinear chains $12,12 \mid 34$, $125 \mid 34$ and $34,12|34,125| 34$. These two chains are clearly not of the same type. The fact that the functor $X \rightarrow$ set of maximal chains of multisets over $X$, is not analytic, but has the desired multilinear component, brought us to find the analytic functor corresponding to this multilinear component, knowing by [7] that it exists and is unique (more precisely, each analytic functor is obtained by specialization of its multilinear component, another way of expressing Weyl duality). It turned out quickly that the correct functor had to distinguish between blocks of the partition of the multiset, in order to record the history of their formation through joins, starting from the atoms (cf. the previous example); in other words, one was lead naturally to increasing trees, where the label of each internal node records the history of the block under it. In particular, this record is not necessary for multilinear chains, implying the bijection between maximal chains of partitions of a set and increasing trees (see below).

A multilinear tree is a tree such that each element of $X$ appears exactly once as label. To each multilinear tree $t$ in $S(X)$, of degree $n$, we associate a maximal chain of partitions of $X$. This mapping $h(t)$ is described in figure 4 , and the reader will convice himself that it is a bijection.

Denote by $\mathcal{C}(X)$ the $\mathbb{Q}$-vector space spanned by the (maximal) chains of partitions of $X$, and by $\mathcal{S}_{\text {mult }}(X)$ (resp. $\mathcal{S}_{\text {mult }}^{\prime}(X)$ ) the multilinear part of (= span of multilinear trees) $\mathcal{S}(X)$ (resp. $\mathcal{S}^{\prime}(X)$ ). Then $h$ extends to a vector space isomorphism $\mathcal{S}_{\text {mult }}(X) \rightarrow \mathcal{C}(X)$.

Corollary 3.2 $\mathcal{R}_{\text {mult }}(X)$ is naturally isomorphic with the quotient of $\mathcal{C}(X)$ by the cohomology (or Garsia-Stanton) relations, i.e., the subspace $\mathcal{C}^{\prime}(X)$ generated by the elements 
of the form

$$
\begin{aligned}
& (\ldots, \ldots A|B| C|D \ldots \ldots, \ldots \cup B| C|D \ldots \ldots, A \cup B| C \cup D \ldots \ldots) \\
& \quad+(\ldots, \ldots A|B| C|D \ldots, \ldots A| B|C \cup D \ldots \ldots A \cup B| C \cup D \ldots \ldots) \\
& (\ldots, \ldots A|B| C \ldots, \ldots A \cup B \mid C \ldots, \ldots A \cup B \cup C \ldots, \ldots) \\
& \quad+(\ldots, \ldots A|B| C \ldots, \ldots B \cup C \mid A \ldots, \ldots A \cup B \cup C \ldots, \ldots) \\
& \quad+(\ldots, \ldots A|B| C \ldots, \ldots C \cup A \mid B \ldots, \ldots A \cup B \cup C \ldots, \ldots)
\end{aligned}
$$

This result has also been obtained independently by Michelle Wachs [14].

Proof: The corollary is simply a translation of Theorem 3.1, via the bijection $h$; indeed, it implies that $\mathcal{R}_{\text {mult }}(X) \simeq \mathcal{S}_{\text {mult }}(X) / \mathcal{S}_{\text {mult }}^{\prime}(X)$.

We now show how to compute directly the previous isomorphism. For this, recall that the lattice $\pi(X)$ of partitions of $X$ is generated by its atoms; such an atom will be denoted by $a=(x y)$, for $x, y$ in $X$. Furthermore, each chain may be written $c=\left(0, a_{1}, a_{1}\right.$ $\wedge a_{2}, \ldots, a_{1} \wedge a_{2} \wedge \cdots \wedge a_{n-1}=1$ ), where 0 (1) is the partition with $n$ blocks (one block) and $n=|X|$. We write $\underline{a}=\left(a_{1}, \ldots, a_{n-1}\right)$ and $c(\underline{a})=c$. For such an $\underline{a}$ and $\sigma \in S_{n-1}$, let $\underline{a} \cdot \sigma=\left(a_{\sigma_{1}}, \ldots, a_{\sigma(n-1)}\right)$. For a multilinear word $w$ on $X, w=x_{1}, \ldots, x_{n}$, let $\underline{a}(w)=\left(\left(x_{1} x_{2}\right),\left(x_{2} x_{3}\right), \ldots,\left(x_{n-1} x_{n}\right)\right)$.

Corollary 3.3 Let $\mathcal{R}(X)$ be embedded in $\mathbb{Q}\langle X\rangle$. The isomorphism $P: \mathcal{C}(X) / \mathcal{C}^{\prime}(X) \rightarrow$ $\mathcal{R}_{\text {mult }}(X)$ of Corollary 3.2 is given by

$$
P(c)=\sum_{w, \alpha}(-1)^{\alpha} w
$$

where the summation is over all multilinear words $w$ on $X, n=|X|$, and all $\alpha$ in $S_{n-1}$ such that $c=c(\underline{a}(w) \cdot \alpha)$.

This formula has been given in [11].

Proof: Let $c$ be a fixed chain on $X$. There is a bijection between the set $\{t \in T(X) \mid$ $h g(t)=c\}$ and $\left\{(w, \alpha) \mid w \in X_{\text {mult }}^{*}, \alpha \in S_{n-1}, c=c(\underline{a}(w) \cdot \alpha)\right\}$ given by: $w=w(t), \alpha=$ $\sigma(t)^{-1}$, where $\sigma(t)$ is the permutation of $t$. By looking at figure 5, the reader will convince himself that it is indeed a bijection, and that $\alpha=\sigma^{-1}$. Since the previous set of trees is by definition of a spatial tree a single class mod. $\sim$, this implies the corollary, in view of Corollaries 2.3 and 3.2.

\section{Spatial trees}

An element of $V(X)$ will be a spatial tree $\tau$ with vertices labelled in $X$, and edges having distinct labels in $\{1,2, \ldots, n-1\}$, where $\tau$ has $n$ vertices. There is a natural mapping from 


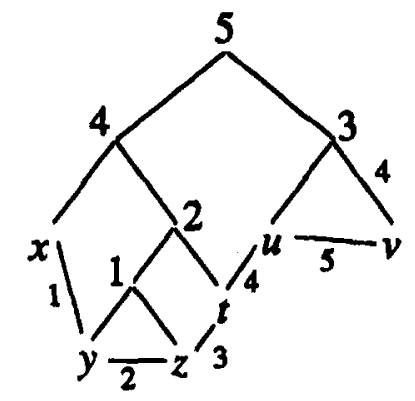

$$
\begin{aligned}
& \sigma=41253 \\
& \alpha=23514 \\
& w=x y z t u v
\end{aligned}
$$

Figure 5.
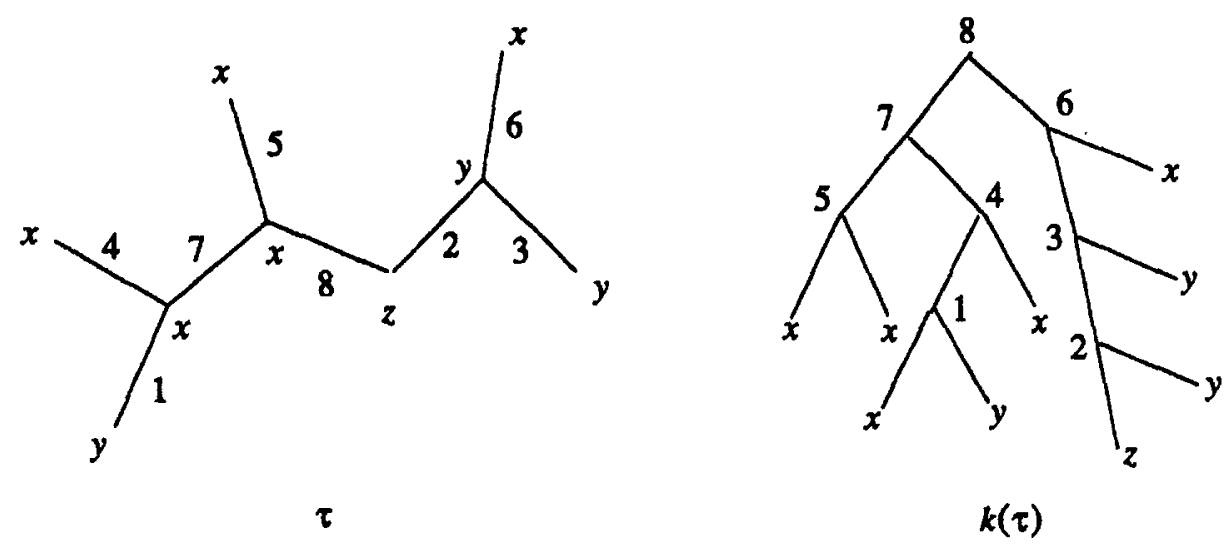

Figure 6.

$V(X)$ into $S(X)$, described in figure 6 . The recursive definition of $k$ goes as follows: if $\tau$ has one vertex labelled $x$, then $k(\tau)$ is the tree reduced to a leaf labelled $x$; if $\tau$ has $n$ vertices, then the edge with the maximal label $n-1$ has two subtrees $\tau_{1}$ and $\tau_{2}$, and $k(\tau)$ has a root labelled $n-1$ with the two immediate subtrees $k\left(\tau_{1}\right)$ and $k\left(\tau_{2}\right)$.

Denote by $\mathcal{V}(X)$ the $\mathbb{Q}$-linear span of $V(X)$. For $\tau$ as before and $\alpha \in S_{n-1}$, denote by $\tau \cdot \alpha$ the tree in $V(X)$ obtained by replacing each edge label $i$ by $\alpha^{-1}(i)$; this is a right action of $S_{n-1}$ on $V(X)$.

Define a pairing between $V(X)$ and $\mathcal{S}(X)$ by

$$
\langle\tau, t\rangle=\sum_{\alpha \in S_{n-1}}(-1)^{\alpha},
$$

where the sum is over the $\alpha$ such that $k(\tau \cdot \alpha)=t$.

Define a subspace $\mathcal{V}^{\prime}(X)$ of $\mathcal{V}(X)$, generated by elements of the form shown in figure 7 , where dots indicate that the trees are otherwise equal. 

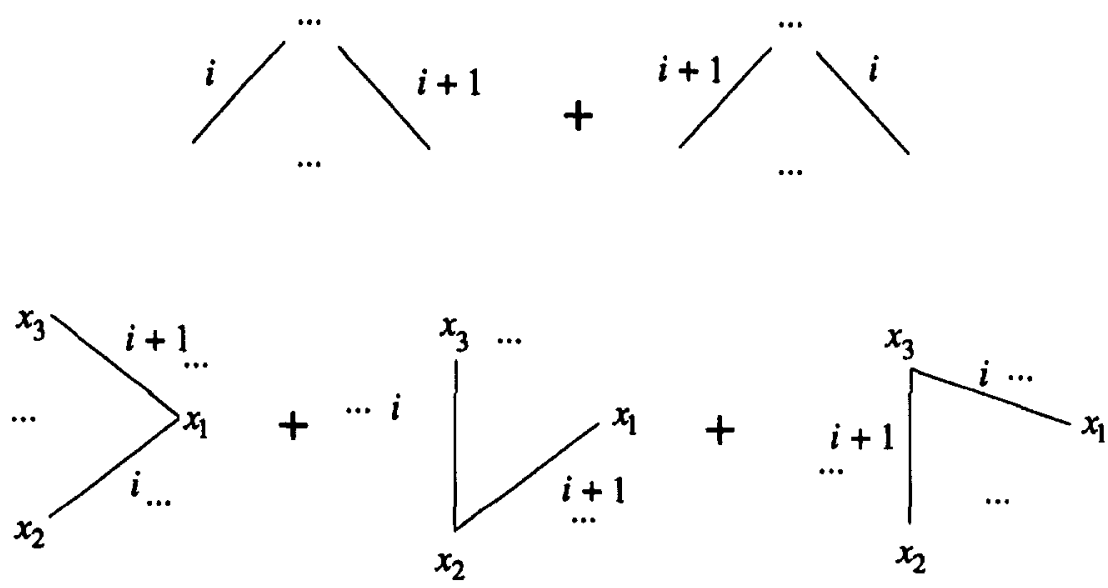

Figure 7.

$$
\begin{gathered}
x_{1} \stackrel{1}{-} x_{2} \stackrel{2}{2} x_{3} \cdots \quad \frac{n-1}{\tau_{w}} x_{n} \\
\end{gathered}
$$

Figure 8.

Theorem 4.1 The pairing (4.1) induces a duality between $\mathcal{V}(X) / \mathcal{V}^{\prime}(X)$ and $\mathcal{S}(X) / \mathcal{S}^{\prime}(X)$. Hence, the first space is naturally isomorphic with the dual of the free Lie super algebra $\mathcal{R}(X)$.

The proof of Theorem 4.1 is a consequence of several lemmas, which will be proved in the last section.

Lemma 4.2 If $T$ is in $\mathcal{V}^{\prime}(X)$ and $t$ in $S$, then $\langle T, t\rangle=0$. If $\tau$ is in $V$ and $T$ in $\mathcal{S}^{\prime}(X)$, then $\langle\tau, T\rangle=0$.

If $w=x_{1} \cdots x_{n}$ is a word of length $n$ in $X^{*}$, let $\tau_{w}$ denote the element of $V(X)$ shown in figure 8. Denote by $\langle P, w\rangle$ the coefficient of $w$ in $P \in \mathbb{Q}\langle X\rangle$. Denote by $P(t)$ the Ree polynomial associated to $t \in S(X)$ through the mapping of Theorem 3.1.

Lemma 4.3 For any $w$ in $X^{*}$ and $t$ in $S(X)$, one has $\left\langle\tau_{w}, t\right\rangle=\langle P(t), w\rangle$.

Lemma 4.4 Let $x_{0} \in X$. Then each element of $V(X)$ involving $x_{0}$ is equal mod. $\mathcal{V}^{\prime}(X)$ to a linear combination of $\tau_{x_{0} w}, w \in X^{*}$. 
Proof of Theorem 4.1: Lemma 4.2 shows that the pairing (4.1) induces a pairing between $\mathcal{V} / \mathcal{V}^{\prime}$ and $\mathcal{S} / \mathcal{S}^{\prime}$. We know by Theorem 3.1 that $\mathcal{S} / \mathcal{S}^{\prime}$ is isomorphic with $\mathcal{R}(X)$. Moreover, this space is embedded in $\mathbb{Q}(X)$, so the linear functions on $\mathcal{R}(X)$ of the form $P \mapsto$ $\langle P, w\rangle, w \in X^{*}$, span the dual of $\mathcal{R}(X)$. Thus, Lemma 4.3 implies that the pairing is nondegenerate on the right, i.e., if for $T$ in $\mathcal{S}$, one has $\langle\tau, T\rangle=0$ for any $\tau$ in $V$, then $T \in \mathcal{S}^{\prime}$.

Restricting to multilinear elements, we see that each linear form on $\mathcal{R}_{\text {mult }}(X)=\mathcal{S}_{\text {mult }}$ $(X) / \mathcal{S}_{\text {mult }}^{\prime}(X)$ is in $\mathcal{V}_{\text {mult }} / \mathcal{V}_{\text {mult }}^{\prime}$; since $\mathcal{R}_{\text {mult }}(X)$ is of dimension $(|X|-1)$ !, we deduce that $\mathcal{V}_{\text {mult }} / \mathcal{V}_{\text {mult }}^{\prime}$ is of dimension $\geq(|X|-1)$ !. Lemma 4.4 shows that its dimension is $\leq(|X|-1)$ !. Thus the pairing between $\mathcal{S}_{\text {mult }} / \mathcal{S}_{\text {mult }}^{\prime}$ and $\mathcal{V}_{\text {mult }} / \mathcal{V}_{\text {mult }}^{\prime}$ is a duality.

From this, we deduce that if $\varphi$ is a linear form on $\mathcal{V}_{\text {mult }}$ which vanishes on $\mathcal{V}_{\text {mult }}^{\prime}$, then it is of the form $\varphi(T)=\left\langle T, T^{\prime}\right\rangle$ for some $T^{\prime}$ in $\mathcal{S}_{\text {mult. }}$ Let $Q: \mathbb{Q}(X) \rightarrow \mathcal{V}(X)$ be the linear mapping sending $w$ on $\tau_{w}$. Then, by Lemma 4.3, for $u, v$ in $X^{+}$such that their signed shuffle product $u \times v$ (cf. [12]) is multilinear, we have $\varphi(Q(u \times v))=\left\langle Q(u \times v), T^{\prime}\right\rangle=\left\langle P\left(T^{\prime}\right), u \times v\right\rangle=0$, since $P\left(T^{\prime}\right)$ is a Ree polynomial.

Thus, each linear form on $\mathcal{V}_{\text {mult }}$ which vanishes on $\mathcal{V}_{\text {mult }}^{\prime}$, vanishes on the multilinear $Q(u \times v)$. We deduce that each such element is in $\mathcal{V}_{\text {mult }}^{\prime}$. By specialization, we have also in general $Q(u \times v) \in \mathcal{V}^{\prime}(X)$.

Now, let $\varphi$ be a linear form on $\mathcal{V}$ which vanishes on $\mathcal{V}^{\prime}$. Let $P=\sum_{w \in X^{*}} \varphi\left(\tau_{w}\right) w$.

Then $\langle P, u \times v\rangle=\sum_{w \in X^{*}} \varphi\left(\tau_{w}\right)\langle w, u \times v\rangle=\varphi\left(\sum_{w}\langle u \times v, w\rangle \tau_{w}\right)=\varphi(Q(u \times v))=0$. Hence, by [12], $P$ is a Ree polynomial, and we find $T^{\prime} \in \mathcal{S}$ such that $P\left(T^{\prime}\right)=P$.

By Lemma 4.3, $\varphi\left(\tau_{w}\right)=\langle P, w\rangle=\left\langle P\left(T^{\prime}\right), w\right\rangle=\left\langle\tau_{w}, T^{\prime}\right\rangle$; since the $\tau_{w}$ span $\mathcal{V}$ mod. $\mathcal{V}^{\prime}$ by Lemma 4.4, we deduce that each linear form on $\mathcal{V} / \mathcal{V}^{\prime}$ is of the form $\left\langle, T^{\prime}\right\rangle$, and the pairing is also nondegenerate on the left.

Let $L$ be the set of Lyndon words in $X^{*}$.

Corollary 4.5 The $\tau_{\ell}$, for $\ell$ in $L$, and the $\tau_{\ell \ell}$ for $\ell$ in $L,|\ell|$ odd, form a basis of $\mathcal{V}(X) / \mathcal{V}^{\prime}(X)$.

Proof: This follows, because the functions $P \mapsto\langle P, l\rangle$, with $\ell$ Lyndon word, and $P \mapsto$ $\langle P, \ell \ell\rangle, \ell \in L,|\ell|$ odd, form a basis of $\mathcal{R}(X)^{*}$.

In the course of the proof of Theorem 4.1, we have also proved the following result.

Corollary 4.6 The elements $\tau_{u \times v}$ are in $\mathcal{V}^{\prime}(X)$, for any non empty words $u, v$.

Direct proofs Corollaries 4.5 and 4.6 would be interesting.

Recall from [9] that the Arnold algebra $A=A(\ell)$ is generated by the elements $a_{i j}=$ $a_{j i}, 1 \leq i, j \leq \ell, i \neq j$, subject to the relations

$$
\begin{aligned}
a_{i j} a_{k m} & =-a_{k m} a_{i j}, \\
a_{i j} a_{j k}+a_{j k} a_{k i}+a_{k i} a_{i j} & =0 .
\end{aligned}
$$

It is shown in [9] p. 415, that $A$ has a highest homogeneous component $A^{\ell-1}$, spanned by the elements $m(\tau)$, which is the product $a_{i_{1} j_{1}} \cdots a_{i_{t-1} j_{\ell-1}}, \tau$ a tree with vertices $\{1, \ldots, \ell\}$ 
and edges $\left\{\left(i_{1}, j_{1}\right), \ldots,\left(i_{\ell-1}, j_{\ell-1}\right)\right\}$. The similarity between (4.2), (4.3) and (4.4), (4.5) implies the following result, proved by Lehrer and Solomon [9].

Corollary 4.7 As an $S_{\ell-\text { module, }} A^{\ell-1}$ is naturally isomorphic with the multilinear part of the dual of $\mathcal{R}(X), X=\{1,2, \ldots, \ell\}$.

\section{Proof of the lemmas}

Proof of Lemma 2.2 We prove first a claim. For $t$ in $T(X)$, let $f_{1}(t)$ denote the tree obtained from $t$ by forgetting the internal labels.

$$
\begin{aligned}
& \text { If } f_{1}(s)=f_{1}(t) \text { and if } \alpha, \sigma \text { are the permutations of } s, t \\
& \text { then }(-1)^{\alpha} s \equiv(-1)^{\sigma} t \bmod . \mathcal{T}^{\prime}(X)
\end{aligned}
$$

Indeed, let $L(s), R(s)$ denote the set of internal labels of the left, right subtree of $s$, with $|L(s)|=i$.

Suppose that $L(s)=\{1, \ldots, i\}, R(s)=\{i+1, \ldots, i+j\}$. If moreover $L(s)=L(t)$, $R(s)=R(t)$, then $s=(-1)^{j}\left[s^{\prime}, s^{\prime \prime}\right], t=(-1)^{j}\left[t^{\prime}, t^{\prime \prime}\right]$ for some $s^{\prime}, s^{\prime \prime}, t^{\prime}, t^{\prime \prime}$ in $T(X)$. Then, we have $f_{1}\left(s^{\prime}\right)=f_{1}\left(t^{\prime}\right), f_{1}\left(s^{\prime \prime}\right)=f_{1}\left(t^{\prime \prime}\right)$ and by induction $(-1)^{\alpha^{\prime}} s^{\prime} \equiv(-1)^{\sigma^{\prime}} t^{\prime}$, $(-1)^{\alpha^{\prime \prime}} s^{\prime \prime} \equiv(-1)^{\sigma^{\prime \prime}} t^{\prime \prime}$ mod. $\mathcal{T}^{\prime}(X)$, where $\alpha^{\prime}, \alpha^{\prime \prime}, \sigma^{\prime}, \sigma^{\prime \prime}$ are the permutations of $s^{\prime}, s^{\prime \prime}$, $t^{\prime}, t^{\prime \prime}$. Since the product in $\mathcal{T}(X)$ is compatible with the quotient mod. $\mathcal{T}^{\prime}(X)$, and since the permutations of $s$ and $t$ are $\alpha=\alpha^{\prime}(i+j+1) \bar{\alpha}^{\prime \prime}, \sigma=\sigma^{\prime}(i+j+1) \bar{\sigma}^{\prime \prime}$ of signature $(-1)^{\alpha^{\prime}}(-1)^{\alpha^{\prime \prime}}(-1)^{j}$ and $(-1)^{\sigma^{\prime}}(-1)^{\sigma^{\prime \prime}}(-1)^{j}$, we obtain $(-1)^{\alpha} s \equiv(-1)^{\sigma} t$, in this case.

If $L(t)$ is not the interval $\{1, \ldots, i\}$, we show by induction on the sum of the elements in $L(t)$, that the claim holds for $t$. If this sum is $i(i+1) / 2, L(t)=\{1, \ldots, i\}$ and the claim holds. Otherwise, this sum is $>i(i+1) / 2$ and we can find $k \notin L(t), k+1 \in L(t)$; then $k+1$ (resp. $k$ ) is in the left (resp. right) subtree of $t$, and if we exchange them, we obtain by (2.3) a tree $t_{1} \equiv-t$. Then $k \in L\left(t_{1}\right)$ and the sum for $t_{1}$ is smaller than the sum for $t$, and their permutations have opposite sign, which concludes this case.

Suppose now that $s$ is not of the form indicated previously. Then the latter argument shows that we can find $s_{1}$ of this form, such that $s_{1}, s$ satisfy the claim; since $s_{1}, t$ also do, so do $t$ and $s$.

The claim being proved, we can now deduce that the product satisfies the super antisymmetry relation. Let $t_{1}, t_{2}$ be two trees of degree $n_{1}, n_{2}$. Then $\left[t_{1}, t_{2}\right]=(-1)^{n_{2}-1} t,\left[t_{2}, t_{1}\right]=$ $(-1)^{n_{1}-1} t^{\prime}$, where $t=\left(t_{1}, \bar{t}_{2}\right), t^{\prime}=\left(t_{2}, \bar{t}_{1}\right)$, with $\bar{t}_{1}, \bar{t}_{2}$ the trees whose internal labels have been appropriately shifted. Let $s=\left(\bar{t}_{1}, t_{2}\right)$; by $(2.5)$ we have $t^{\prime} \equiv s$ and $s$ and $t$ only differ by their permutations, which are obtained one from the other by multiplying it by the permutation $n_{1} \cdots\left(n_{1}+n_{2}-1\right) 1 \cdots\left(n_{1}-1\right)$, of signature $(-1)^{\left(n_{1}-1\right)\left(n_{2}-1\right)}$. Thus, the claim shows that $t \equiv s(-1)^{\left(n_{1}-1\right)\left(n_{2}-1\right)}$, which implies $\left[t_{1}, t_{2}\right] \equiv(-1)^{\left(n_{2}-1\right)}(-1)^{\left(n_{1}-1\right)\left(n_{2}-1\right)}(-1)^{\left(n_{1}-1\right)}$ $\left[t_{2}, t_{1}\right]=(-1)^{n_{1} n_{2}-1}\left[t_{2}, t_{1}\right]$, what was to be shown.

Let $P, Q, R$ be three trees in $T(X)$ coded as words in $L(X)$, according to the bijection of Section 2. We have to show that $[P,[Q, R]]-[[P, Q], R]-(-1)^{p q}[Q,[P, R]]$ is in $\mathcal{T}^{\prime}(X)$. We denote by $P_{i j}$ the tree $P$ where the internal labels have been shifted to the interval $[i, j]$ (with $j-i+1=|P|-1$ equal to the number of internal nodes of $P$ ). Then, 
by the definition of the product, the above element is

$$
\begin{aligned}
& (-1)^{r-1}(-1)^{q+r-1} P(p+q+r-1) Q_{p, p+q-2}(p+q+r-2) R_{p+q-1, p+q+r-3} \\
& \quad-(-1)^{q-1}(-1)^{r-1} P(p+q-1) Q_{p, p+q-2}(p+q+r-1) R_{p+q, p+q+r-2} \\
& -(-1)^{p q}(-1)^{r-1}(-1)^{p+r-1} Q(p+q+r-1) P_{q, p+q-2} \\
& \quad \times(p+q+r-2) R_{p+q-1, p+q+r-3} .
\end{aligned}
$$

We know that the element

$$
\begin{aligned}
& P(p+q+r-1) Q_{p, p+q-2}(p+q+r-2) R_{p+q-1, p+q+r-3} \\
& \quad+P(p+q+r-2) Q_{p, p+q-2}(p+q+r-1) R_{p+q-1, p+q+r-3} \\
& \quad+Q_{p, p+q-2}(p+q+r-1) P(p+q+r-2) R_{p+q-1, p+q+r-3}
\end{aligned}
$$

is in $\mathcal{T}^{\prime}(X)$ : indeed, it is obtained with $i=p+q+r-2$ in (2.4), by using (2.5). So, by the claim, all we have to show is that the permutation which sends $p+q-1$ onto $p+q+r-2$ and sends the interval $[p+q, p+q+r-2]$ onto $[p+q-1, p+q+r-3]$ (resp. sends the interval $[1, q-1]$ onto $[p, p+q-2]$ and the interval $[q, p+q-2]$ onto $[1, p-1])$, leaving fixed the other digits, has signature $(-1)^{r-1}$ (resp. $(-1)^{p q+p+q-1}$ ). This verification is left to the reader.

\section{Proof of Lemma 4.2:}

1. We have to show that for $T=(4.2)$ or $(4.3),\langle T, t\rangle=0$.

This is clear for (4.2), since the transposition $(i, i+1)$ is of signature -1 , and $\tau_{1}$. $(i, i+1)=\tau_{2}$, where $\tau_{1}, \tau_{2}$ are the two trees in (4.2).

Let $\tau_{1}, \tau_{2}, \tau_{3}$ be the three trees in (4.3) and let $t \in S(X)$. We have to show that $\sum_{1 \leq j \leq 3} \sum_{k\left(\tau_{j}, \alpha\right)=t}(-1)^{\alpha}=0$. Suppose that $k\left(\tau_{j} \cdot \alpha\right)=t$, and let $r=\alpha^{-1}(i), s=\alpha^{-1}$ $(i+1)$. Suppose that $r<s$. We take the indices $j$ mod. 3 . Then $\tau_{j}, \tau_{j} \cdot \alpha, \tau_{j+1}, \tau_{j+1} \cdot(i$, $i+1) \alpha$ and $t$ are shown in figure 9. Hence $t=k\left(\tau_{j} \cdot \alpha\right)=k\left(\tau_{j+1} \cdot(i, i+1) \alpha\right)$. Similarly, if $r>s$, then $t=k\left(\tau_{j-1} \cdot(i, i+1) \alpha\right)$. This implies the vanishing of the previous sum.

2. Let $\tau \in V(X)$. If $k(\tau \cdot \alpha)$ is equal to the tree $t_{1}$ at the left in $(2.3)$, then $k(\tau \cdot(i, i+1) \alpha)$ is equal to the tree $t_{2}$ on the right. Hence $\left\langle\tau, t_{1}+t_{2}\right\rangle=0$.

For (2.4), denote by $t_{1}, t_{2}, t_{3}$ the three trees. We have to show that $\sum_{1 \leq j \leq 3} \sum_{k(\tau \cdot \alpha)=t_{j}}$ $(-1)^{\alpha}=0$. Suppose that $k(\tau \cdot \alpha)=t_{j}$. We take the indices $\bmod 3$. Then, by construction of the mapping $k$, the subtree of $\tau$ corresponding to $A_{j+2}$ is connected by the edge labelled $\alpha(i+1)$ either to the subtree corresponding to $A_{j+1}$ or the one corresponding to $A_{j}$. Figure 10 shows $\tau, \tau \cdot \alpha, \tau \cdot \alpha o(i, i+1)$. In that case, we have $k(\tau \cdot \alpha o(i, i+1))=t_{j+1}$. In the second case, similarly, $k\left(\tau \cdot \alpha(i, i+1)=t_{j-1}\right.$. This proves that the previous sum vanishes. 

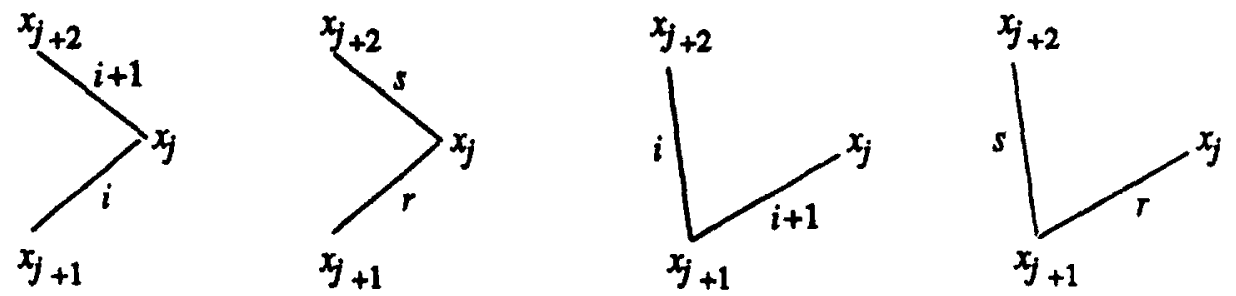

$\tau_{j}$

$$
\tau_{j} \cdot \alpha
$$

$\tau_{j+1}$

$\tau_{j+1} \cdot(i, i+1) \alpha$

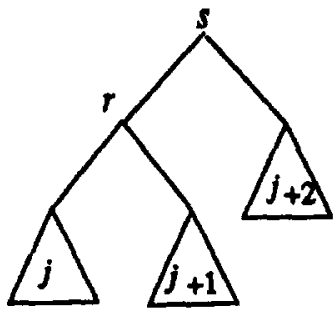

$t$

Figure 9.

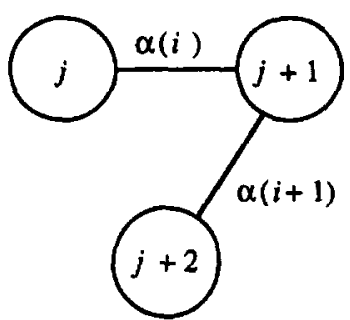

$\tau$

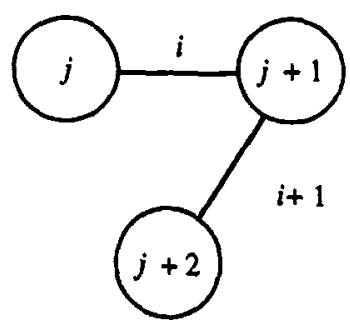

$\tau \cdot \alpha$

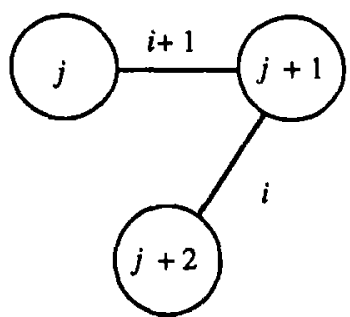

$\tau \cdot \alpha \circ(i, i+1)$

Figure 10.

Proof of Lemma 4.3: Let $w$ be a fixed word of length $n$ in $X^{*}, w=x_{1} \cdots x_{n}$; there is a bijection $\varphi$ from $S_{n-1}$ onto $\left\{t^{\prime} \in T(X) \mid w\left(t^{\prime}\right)=w\right\}$, such that $t^{\prime}=\varphi(\alpha)$ implies that $g\left(t^{\prime}\right)=k\left(\tau_{w} \cdot \alpha\right)$ and $\alpha=\sigma\left(t^{\prime}\right)^{-1}$. Suppose that the existence of $\varphi$ is proved. Then the condition: $t^{\prime} \in T(X), w\left(t^{\prime}\right)=w, g\left(t^{\prime}\right)=t$ implies that there exists $\alpha \in S_{n-1}$ with $\varphi(\alpha)=t^{\prime}$, $t=g\left(t^{\prime}\right)=k\left(\tau_{w} \cdot \alpha\right)$ and $\alpha$ and $\sigma\left(t^{\prime}\right)$ have the same signature; conversely, $\alpha \in S_{n-1}$ and $k\left(\tau_{w} \cdot \alpha\right)=t$ implies that $t^{\prime}=\varphi(\alpha)$ satisfies $g\left(t^{\prime}\right)=t$ and $w\left(t^{\prime}\right)=w$. Hence $\left\langle\tau_{w}, t\right\rangle=$ $\sum(-1)^{\alpha}$ (sum over $\alpha \in S_{n-1}$ with $\left.k\left(\tau_{w} \cdot \alpha\right)=t\right)=\sum(-1)^{\sigma\left(t^{\prime}\right)}$ (sum over $t^{\prime} \in T(X)$ with $g\left(t^{\prime}\right)=t$ and $\left.w\left(t^{\prime}\right)=w\right)=\langle P(t), w\rangle$, the latter equality by Corollary 2.3. 


$$
\begin{aligned}
& w=x \quad x \quad y \quad x \quad y \\
& \tau_{w}=x-x-y-x-y \\
& \alpha=3 \quad 1 \quad 4 \quad 2 \\
& \tau_{w} \cdot \alpha=x-x-y-x-y \\
& \sigma\left(t^{\prime}\right)=2 \quad 4 \quad 1 \quad 3
\end{aligned}
$$

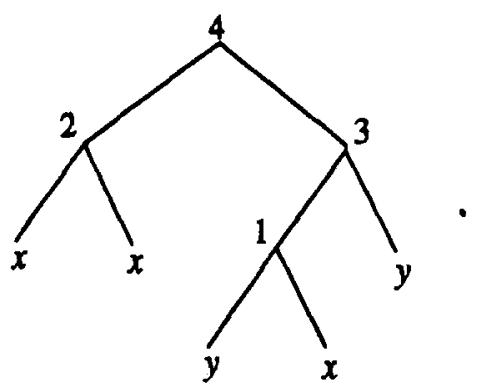

$t^{\prime}=\varphi(\alpha)$

Figure 11.

The construction of $\varphi$ is a variant of that of $k$, illustrated in figure 11. The fact that it is a bijection is left to the reader.

Proof of Lemma 4.4: First, we show that if $\tau$ involves $x_{0}$, then $\tau$ is a linear combination of $\tau^{\prime}$ involving $x_{0}$ as an end-point. If $x_{0}$ is not an end-point in $\tau$, then $x_{0}$ is a point of order $n \geq 2$, and we take $x_{1}=x_{0}$ in figure 7 . Hence $\tau$ is mod. $\mathcal{V}^{\prime}$ a linear combination of $\tau^{\prime}$, involving $x_{0}$ at order $n-1$, and we conclude by induction.

Now, we modify slightly an argument of [9] p. 415.

Now $\tau$ involves $x_{0}$ as an end-point. Call tail of $\tau$ the maximal subtree containing $x_{0}$, which has no vertex of order $\geq 3$. If $\tau$ is not a chain, let $x_{1}$ be the vertex not in the tail, neighbour of the end-point of the tail distinct from $x_{0}$. Then, using repeatedly (4.2), we may suppose that $\tau$ is the left tree (4.3), with $x_{2}, x_{3}$ not in the tail, and $x_{1}$ of order $n \geq 3$. In the two other trees, either $x_{1}$ is still of order $\geq 3$, but smaller, or they have a longer tail. Hence, we conclude by induction that we may suppose that $\tau$ is a chain.

Then, using (4.2), we can arrange the labels in increasing order, starting from $x_{0}$, which concludes the proof.

\section{References}

1. H. Barcelo, "On the action of the symmetric group on the free Lie algebra and the partition lattice," $J$. Combin. Th. A 55 (1990), 93-129.

2. H. Barcelo and N. Bergeron, "The Orlik-Solomon algebra on the partition lattice and the free Lie algebra," J. Combin. Th. A 55 (1990), 80-92.

3. A. Björner, "On the homology of geometric lattices," Alg. Univ. 14 (1982), 107-128.

4. A. Garsia and D. Stanton, "Group actions on Stanley-Reisner rings and the construction of invariants," Adv. Math. 51 (1984), 107-201.

5. P. Hanlon, "The fixed point partition lattice," Pacific J. Math. 96 (1981), 319-341.

6. P. Hanlon and M. Wachs, "On Lie $k$-algebras," Advances Maths. 113 (1995), 206-236.

7. A. Joyal, "Foncteurs analytiques et especes de structure," Lect. Notes Math. 1234 (1985), 126-159.

8. A.A. Klyachko, "Lie elements in the tensor algebra," Sib. Mat. Zhurnal (translation) 15 (1974), 241-256.

9. G.I. Lehrer and L. Solomon, "On the action of the symmetric group on the cohomology of the complement of its reflecting hyperplanes," J. Algebra, 104 (1986), 410-424. 
10. I.G. Macdonald, Symmetric Functions and Hall Polynomials, Clarendon Press, Oxford, 1979.

11. G. Melançon and C. Reutenauer, "Une présentation combinatoire de la super-algèbre de Lie libre," C.R. Acad. Sci. Paris, Sér. I 1315 (1992), 1215-1220.

12. R. Ree, "Generalized Lie elements," Can. J. Math. 12 (1960), 493-502.

13. R. Stanley, "Some aspects of groups acting on finite posets," J. Combin. Th. A 32 (1982), 132-161.

14. M. Wachs, "Cohomology of partition Jattices and free Lie algebras," Discrete Maths, to appear. 